\title{
Video Article \\ Slice Preparation, Organotypic Tissue Culturing and Luciferase Recording of Clock Gene Activity in the Suprachiasmatic Nucleus
}

\author{
Sergey A. Savelyev ${ }^{* 1}$, Karin C. Larsson ${ }^{* 1}$, Anne-Sofie Johansson ${ }^{1}$, Gabriella B. S. Lundkvist ${ }^{1}$ \\ ${ }^{1}$ Swedish Medical Nanoscience Center, Department of Neuroscience, Karolinska Institutet \\ *These authors contributed equally
}

Correspondence to: Gabriella B. S. Lundkvist at gabriella.schmitz.lundkvist@ki.se

URL: https://www.jove.com/video/2439

DOI: doi:10.3791/2439

Keywords: Neuroscience, Issue 48, suprachiasmatic nucleus, mice, organotypic tissue culture, circadian rhythm, clock gene, Period 2, luciferase

Date Published: 2/15/2011

Citation: Savelyev, S.A., Larsson, K.C., Johansson, A.S., Lundkvist, G.B. Slice Preparation, Organotypic Tissue Culturing and Luciferase Recording of Clock Gene Activity in the Suprachiasmatic Nucleus. J. Vis. Exp. (48), e2439, doi:10.3791/2439 (2011).

\section{Abstract}

A central circadian ( $24 \mathrm{hr}$ ) clock coordinating daily rhythms in physiology and behavior resides in the suprachiasmatic nucleus (SCN) located in the anterior hypothalamus. The clock is directly synchronized by light via the retina and optic nerve. Circadian oscillations are generated by interacting negative feedback loops of a number of so called "clock genes" and their protein products, including the Period (Per) genes. The core clock is also dependent on membrane depolarization, calcium and CAMP ${ }^{1}$. The SCN shows daily oscillations in clock gene expression, metabolic activity and spontaneous electrical activity. Remarkably, this endogenous cyclic activity persists in adult tissue slices of the SCN $2-4$. In this way, the biological clock can easily be studied in vitro, allowing molecular, electrophysiological and metabolic investigations of the pacemaker function.

The SCN is a small, well-defined bilateral structure located right above the optic chiasm ${ }^{5}$. In the rat it contains $~ 8.000$ neurons in each nucleus and has dimensions of approximately $947 \mu \mathrm{m}$ (length, rostrocaudal axis) $\times 424 \mu \mathrm{m}$ (width) $\times 390 \mu \mathrm{m}$ (height) ${ }^{6}$. To dissect out the SCN it is necessary to cut a brain slice at the specific level of the brain where the SCN can be identified. Here, we describe the dissecting and slicing procedure of the SCN, which is similar for mouse and rat brains. Further, we show how to culture the dissected tissue organotypically on a membrane ${ }^{7}$, a technique developed for SCN tissue culture by Yamazaki et al. ${ }^{8}$. Finally, we demonstrate how transgenic tissue can be used for measuring expression of clock genes/proteins using dynamic luciferase reporter technology, a method that originally was used for circadian measurements by Geusz et al. ${ }^{9}$. We here use SCN tissues from the transgenic knock-in PERIOD2::LUCIFERASE mice produced by Yoo et al. ${ }^{10}$. The mice contain a fusion protein of PERIOD (PER) 2 and the firefly enzyme LUCIFERASE. When PER2 is translated in the presence of the substrate for luciferase, i.e. luciferin, the PER2 expression can be monitored as bioluminescence when luciferase catalyzes the oxidation of luciferin. The number of emitted photons positively correlates to the amount of produced PER2 protein, and the bioluminescence rhythms match the PER2 protein rhythm in vivo ${ }^{10}$. In this way the cyclic variation in PER2 expression can be continuously monitored real time during many days. The protocol we follow for tissue culturing and real-time bioluminescence recording has been thoroughly described by Yamazaki and Takahashi $^{11}$.

\section{Video Link}

The video component of this article can be found at https://www.jove.com/video/2439/

\section{Solution Preparation}

1. Culture medium with air-buffering capacity

1. Fill a 1 liter bottle with approximately $800 \mathrm{~mL}$ sterile $\mathrm{H}_{2} \mathrm{O}$ (autoclaved milliQ $\mathrm{H}_{2} \mathrm{O}$ ).

2. While stirring, add and mix following substances: 1 container low glucose, serum-free DMEM 2902 powder without sodium bicarbonate and phenol red (phenol red interferes with the bioluminescence signal), $20 \mathrm{~mL}$ B27 supplement $50 \mathrm{x}, 4.7 \mathrm{~mL}$ of a $7.5 \% \mathrm{NaHCO}_{3}$ solution (or $0.35 \mathrm{~g} \mathrm{NaHCO}_{3}$ ), $10 \mathrm{~mL}$ HEPES $1 \mathrm{M}, 2.5 \mathrm{~mL}$ PenStrep 10,000 U/ mL and $3.5 \mathrm{~g} \mathrm{D}$-glucose. Let the medium stir until the ingredients are completely dissolved. The final medium (1 liter) will contain: 1x DMEM, 1x B27 supplement, $4.2 \mathrm{mM} \mathrm{NaHCO}$, $10 \mathrm{mM} \mathrm{HEPES,} 25 \mathrm{U} / \mathrm{mL}$ penicillin, $25 \mathrm{U} / \mathrm{mL}$ streptomycin and $19 \mathrm{mM} \mathrm{D-glucose.}$

3. Adjust $\mathrm{pH}$ to 7.2 , using $\mathrm{NaOH}$ to decrease or $\mathrm{HCl}$ to increase the $\mathrm{pH}$, and bring volume up to 1 liter with sterile $\mathrm{H}_{2} \mathrm{O}$.

4. Adjust osmolality with $\mathrm{H}_{2} \mathrm{O}$ (decrease osmolality) or D-glucose (increase osmolality). The osmolality must be between $285-315 \mathrm{mOsm} /$ $\mathrm{Kg}$ but the optimal range is $300-310 \mathrm{mOsm} / \mathrm{Kg}$. Do not to dilute the medium more than $5 \%$.

5. Filter the culture medium using Corning sterile vaccum filtration sets (e.g. $2 \times 500 \mathrm{~mL}$ with pore size $0.22 \mu \mathrm{m}$ ) in a sterile hood. Keep the medium in $4^{\circ} \mathrm{C}$ and protect it from light using aluminum foil. We recommend that the medium is used within 3 months. 
2. Hank's balanced salt solution (HBSS) buffer with supplements (for cutting slices)

1. Fill a 1 liter glass bottle with $\sim 600 \mathrm{~mL}$ sterile (autoclaved milliQ) $\mathrm{H}_{2} \mathrm{O}$ and add the following substances: $100 \mathrm{~mL}$ HBSS $10 x$ stock, 10 $\mathrm{mL}$ Penicillin-Streptomycin $10,000 \mathrm{U} / \mathrm{mL}, 5 \mathrm{~mL}$ of a $7.5 \% \mathrm{NaHCO}_{3}$ solution and $10 \mathrm{~mL}$ HEPES $1 \mathrm{M}$.

The final cutting solution will contain:

1x HBSS, $10 \mathrm{mM}$ HEPES, $4.5 \mathrm{mM} \mathrm{NaHCO}$, $100 \mathrm{U} / \mathrm{mL}$ penicillin and $100 \mathrm{U} / \mathrm{mL}$ streptomycin.

2. Check $\mathrm{pH}$ and, if necessary, adjust $\mathrm{pH}$ to 7.2 and bring the volume up to 1 liter with sterile $\mathrm{H}_{2} \mathrm{O}$.

3. Check osmolality, which must be between $285-315 \mathrm{mOsm} / \mathrm{Kg}$.

4. Cool the HBSS to $4^{\circ} \mathrm{C}$. The HBSS buffer needs to be very cold $\left(4^{\circ} \mathrm{C}\right)$ during the slicing/cutting procedure in order to bring down the metabolism and preserve tissue viability.

\section{Preparations Before Cutting and Culturing Slices}

1. The tissues are cultured in a warm dry chamber without $\mathrm{CO}_{2}$. To avoid drying out the cultures need to be sealed with cover glasses attached to the dishes by grease. For this purpose, fill $5 \mathrm{~mL}$ syringes with silicone-based vacuum grease. Cover the syringe tips with small pieces of aluminum foil and autoclave them. Also, autoclave filter papers (used during slicing procedure).

2. Right before slicing procedure, apply autoclaved vacuum grease on the top ring surface of $35 \mathrm{~mm}$ petri dishes. Prepare a separate petri dish for each slice culture.

3. Before starting slicing procedure all non-sterile instruments, razor blades, vibratome blades, cover glasses and other material used for the slice and culture procedure have to be sterilized. Spray all non-sterile equipment with $70 \%$ ethanol. Expose the instruments and the greased petri dishes with UV in a sterile hood for at least 30 minutes UV exposure before culturing.

4. At the same time, fill a Falcon tube with air-buffering culture medium (count $1.2 \mathrm{~mL}$ for each culture; for 8 cultures prepare for instance $10 \mathrm{~mL}$ medium) and add freshly thawed luciferin ( $0.1 \mathrm{M}$ stock solution; Promega, Madison, WI) $(10 \mu \mathrm{L}$ luciferin to $10 \mathrm{~mL}$ medium; final concentration $0.1 \mathrm{mM})$.

5. The luciferin is light sensitive and both stock solutions and the luciferin-containing medium need to be protected from light. Place the Falcon tube with luciferin-medium in a dark $36-37^{\circ} \mathrm{C}$ heating chamber. If appropriate, the luciferin can also be added directly into the culture dishes at time of cutting. If luciferin is not added, there will be no light reaction between luciferase and luciferin and therefore no signal from the tissue.

6. After UV exposure, attach a sterile blade to the vibratome.

\section{SCN Slicing Procedure}

The following procedure describes slicing of adult, typically 2-4 months old, C57/BL6 mice. Please note: the cutting procedure, as well as light exposure of the animal during the night, can differentially reset the phase of the SCN. To avoid this, the cutting and culturing should be performed during the light hours, preferably between ZT 6-12, when no substantial phase shifts due to the procedure occur ${ }^{12}$. If SCN has to be sampled in darkness, 3.1 and 3.2 have to be performed in red light or with night goggles to avoid light-induced phase shifts.

1. Anesthetize the mouse preferably by isofluorane (Baxter) in a glass chamber. When the animal has lost its pain reflexes (check by pinching with the nails in the paw) but has not yet stopped breathing (to maintain oxygen supply as long as possible), rapidly decapitate the head with a pair of scissors or similar.

Please note: in some countries $\mathrm{CO}_{2}$ exposure (hypercapnia) is still permitted as anesthetic method, although it has been reported to promote animal anxiety. In addition, cervical dislocation may be required before decapitation. Please follow local legislations when euthanizing animals.

2. Remove the eyes from the head with scissors in order to prevent strain and further excitation of the optic nerves, which can damage the SCN

3. If still attached, remove the last cervical vertebrate with scissors, remove the skin (Fig 1a) and make two cuts with a fine pair of scissors (for instance iris scissors), one cut at each side of the skull along the sides, thus making a removable "lid".

4. Open the skull with a micro rongeur tool (fine dissecting scissors can also be used on a mouse) and remove all bone until the olfactory bulbs can be seen. Work upwards, never pressing down the brain with the tool, in order to prevent damage of the ventral SCN (Fig 1b).

5. Carefully cut the optic nerve between the olfactory bulbs and the hemispheres using fine micro dissecting spring scissors. Make sure that the nerve is completely cut since stretching of the optic nerve can lead to damage in the SCN and ruptured slices.

6. Turn the head upside down and let the intact brain fall out (if still attached to the brain, other cranial nerves may need to be cut caudal to the optic nerve) into a container (for instance a glass petri dish $\approx 10 \mathrm{~cm}$ ) filled with $50-100 \mathrm{~mL}$ cold HBSS allowing rapid cooling of the brain. Ideally, the two optic nerves should remain intact (Fig 1c). Keep the brain in the HBSS 30-60 seconds to make sure the brain is cooled.

7. Use a spoon or similar and place the chilled brain, dorsal surface up, on a sterile cutting surface (for example a glass petri dish lid turned upside down). In order to prepare a coronal cut, make a perpendicular cut with sterile razor blades or scalpels between the cerebral hemispheres and the cerebellum, thus removing the cerebellum.

8. Apply superglue on the dry platform belonging to the vibroslicer/vibratome.

9. Pick up the brain (cerebral hemispheres without cerebellum and without olfactory bulbs) by inserting a sharp, curved forceps in the rostral part and carefully dry off the HBSS on a sterile filter paper, still holding the brain with the forceps. (Instead of inserting a forceps, a small piece of sterile filter paper can be used to attach and transfer the brain).

10. Fix the hemispheres onto the glued platform with the rostral tip upwards and ventral surface closest to the cutting blade. Attach the platform in the holder belonging to the vibratome (for instance from Campden Instruments, UK) and immediately fill it up with cold HBSS. If the perpendicular cut is made properly, the hemispheres should stand straight up thus providing a good angle necessary for making a coronal cut containing the bilateral SCN.

11. In order to reach the target SCN area, start cutting off thicker sections $(500-800 \mu \mathrm{m})$ of the hemispheres at high or maximum speed of the vibratome. Moving the blade can be fairly quick in the beginning before reaching the hypothalamus but should be slowed down when the optic chiasm becomes visible (high frequency of the vibrating blade and slow movement horizontally decreases cell damage during slicing thus increasing slice viability). Reduce the sections to $100 \mu \mathrm{m}$ when the optic chiasm becomes larger (wider) and the anterior commissure becomes smaller. In order to acquire a mid-SCN section, work yourself "down" (caudal direction) until the two SCN nuclei start to appear. A 
magnifying glass may be necessary for visualizing the nuclei. Please note: the SCN in the mouse brain is located more caudal of the optic chiasm as compared to the rat brain.

12. When the desired level of SCN has been reached (the SCN will at this point appear as more defined, round or almond shaped structures; Fig 1d; approximately Bregma $-0.46--0.70 \mathrm{~mm}$ for the center region of SCN in mouse ${ }^{13}$, Bregma $-0.92--1.40 \mathrm{~mm}$ for rat ${ }^{14}$ ), cut the SCN section (Fig 1e). Suitable thickness of the slice is for mouse $250 \pm 50 \mu \mathrm{m}$; for rat $350 \pm 50 \mu \mathrm{m}$.

13. Using a soft brush, lift and transfer the SCN slice to a lid from a medium sized petri dish filled with cold HBSS, placed under a dissection microscope or stereoscope. Check under magnification if the bilateral SCN is clearly visible. If the mid-region of the SCN will be chosen (which may not necessarily be the only "optimal" slice level but that we consider is the easiest way to standardize the slicing procedure and reduce variation), it should be clearly seen at least on one side of the slice section. If the cut was too rostral, make another section and check for the SCN under magnification.

\section{Organotypic SCN Culture}

1. In the petri dish lid filled with HBSS, dissect out the bilateral SCN as a square tissue $(\sim 1.5 \mathrm{~mm}$ each side) with a pair of sterile scalpels under a dissecting microscope. A small piece of the optic chiasm will remain attached to the explant, but no other nuclei should be included. Cut as close as possible without removing SCN tissue.

2. If appropriate for the planned experiment, the bilateral SCN can then be cut in half to obtain two unilateral SCN. One unilateral SCN can then be used as control (Fig 2a).

3. Fill $1200 \mu \mathrm{L}$ of the luciferin-medium into a $\approx 35 \mathrm{~mm}$ Petri dish and place a culture membrane (Milli-CM $0.4 \mu \mathrm{m}$, Millipore, Bedford, MA) on top of the liquid surface (2b). The culture medium volume is crucial, as the culture membrane should sit securely on the base of the culture dish, not float or rock in the medium ${ }^{11}$. Make sure there are no air bubbles under the membrane. Avoid unnecessary light exposure when working with luciferin.

4. The explants are small and difficult to pick up. Therefore use a $1000 \mu \mathrm{L}$ pipette + tip to suck the SCN explant into the tip and press it out on the membrane. In case the explant is too big to easily fit into a $1000 \mu \mathrm{L}$ pipette tip (e.g. rat SCN; and mouse bilateral SCN) the tip may be cut with a sterile tool to create a wider opening. Discard excessive HBSS on the membrane with the pipette. For luciferase recording, place only one SCN/dish (Fig 2b) as the recording tubes detect all photons emitted from the dish and do not distinguish between signals from different tissues.

5. Seal the dish with a cover glass $\left(\approx 40 \mathrm{~mm}\right.$, Menzel-Gläser, Germany) and vacuum grease (Dow Corning Corp, USA) ${ }^{11}$. Make sure the seal is tight (Fig 2c). If not, seal with more grease.

6. Transfer the dishes to a $36-37^{\circ} \mathrm{C}$ light-tight chamber and start PMT-recordings immediately.

\section{Measurement of Luciferase Activity by Recording Bioluminescence}

Luciferase-induced bioluminescence signals from the small SCN tissue are detected and amplified with photonmultiplier-tube (PMT) detector assemblies mounted inside a light tight chamber. The PMTs are normally positioned $\sim 1-2 \mathrm{~cm}$ above the culture dishes ${ }^{8}$. PMT recording setups can be custom made or are commercially available ${ }^{11}$.

1. Place the dish under a PMT (the heat produced from the PMT will remove condensation on the cover glass) and close the chamber. Make sure the chamber is $100 \%$ light-tight as the dark count of PMTs used for SCN tissues is very low (less than 20 photons/min). The PMTs detect even the weakest light leakage.

2. Start the data acquisition, which is performed with software (for instance LumiCycle; Actimetrics Inc., Wilmette, IL, USA). Photon counts are integrated over 1-10 min intervals to get high resolution of the gene/protein expression.

3. After the recording is finished, the obtained circadian expression of the gene/protein can be analyzed with appropriate software (Origin OriginLab, Northampton, MA, USA; ClockLab, Actimetrics; LumiCycle, Actimetrics Inc; Chrono, Till Roenneberg, University of Munich, Munich, Germany) to determine phase, period (time for one cycle) and amplitude of the rhythm. The peak expression of gene or protein is mostly used as reference point and is defined as the highest photon count during one cycle. The data can be smoothed before analyses of phase, period and amplitude, especially if the signal/noise ratio is low. The baseline sometimes changes and need to be subtracted before the analyses are performed.

\section{Representative Results:}

We here present the oscillatory PER2::LUC expression as a read out for viability and condition of the cultured tissue. Under optimal conditions, and if the tissue is alive, the PER2::LUC expression oscillates with a circadian rhythm as shown in figure 3 . PER2 in the SCN is maximally expressed typically around Zeitgeber Time 12-13 (where ZT 12 represents lights off in a 12:12 hr light:dark cycle). The larger in size the live tissue is, the higher the photon count becomes. However, the size and thickness of organotypically cultured tissue should be kept small in order to keep the tissue viable, preferably not larger than $15 \mathrm{~mm}^{211}$ and not thicker than $500 \mu \mathrm{m}^{7}$. The SCN, if dissected as described here, typically shows photon counts between $10.000-40.000 / \mathrm{min}$ if the tissue is sampled from a homozygous PER2::LUC animal. The amplitude of the oscillation in organotypic SCN cultures is typically very high during the first cycle as compared with the following cycles. It is not entirely clear why the first cycle has very high amplitude. One possible explanation is that a substantial portion of cells in the tissue may die shortly after initial cutting and culturing, thus not utilizing luciferin after the first cycle. The cutting procedure may also cause excessive excitation, which could amplify the luciferase signal during the first cycle.

Figure 3 shows luminescence traces from SCN slices and contains one trace (red) obtained from a slice that was not initially healthy. Dead or unhealthy tissues have low photon count baselines. (In addition, dead tissues often dissociate in the dish and cannot be removed from the membrane in one piece.)

The technique described in this report can beneficially be used in pharmacological experiments. Figure 4 shows a trace from a culture that we treated between day 1 and 2 with a HCN channel blocker (ZD7288, $10 \mu \mathrm{M})$. As can be seen in the figure and as published previously ${ }^{15}$, the 
blocker significantly reduced the amplitude of the circadian oscillation of PER2; however, after washing out with normal culture medium the oscillation came back, demonstrating that the blocker affected the molecular clock but the tissue was viable and healthy.
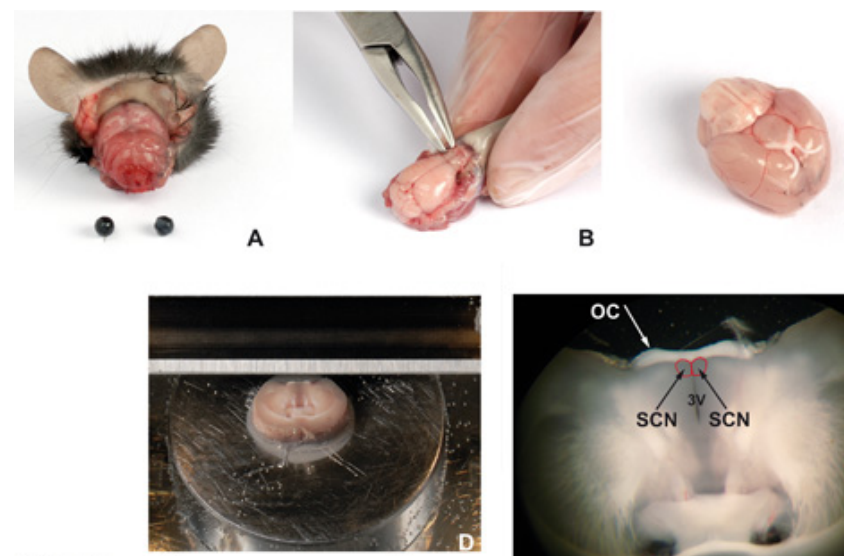

SCN slice procedure

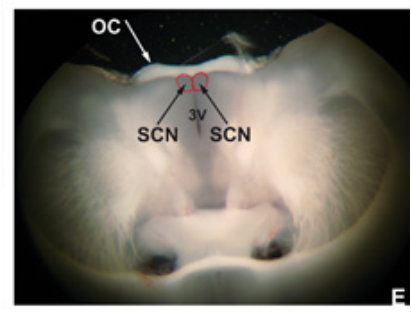

Figure 1. Slicing procedure

A) The head of a euthanized decapitated mouse with eyes and skin removed. B) The skull is removed with a micro rongeur tool. When using the tool, one must work upwards and never press down the brain with the tool. C) The brain shown upside down (ventral side up) without olfactory bulbs. The white optic chiasm with the two intact optic nerves can be seen. The suprachiasmatic nucleus (SCN, the borders marked with red) is located close to the optic chiasm. D) A coronally cut brain attached to the platform in the vibroslicer, at the level of SCN. E) Coronal brain section (250 $\mu \mathrm{m}$ thick) containing the optic chiasm (OC), third ventricle (3V) and the bilateral suprachiasmatic nuclei (SCN).

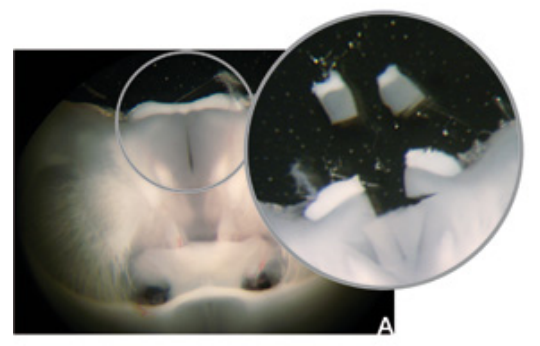

\section{organotypic tissue culture}
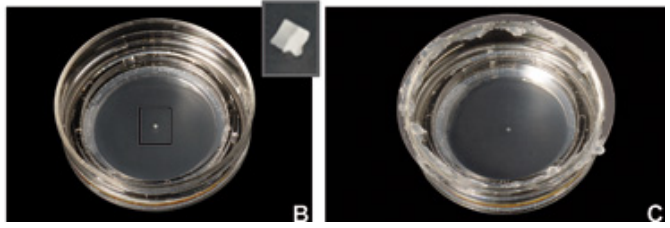

Figure 2. Organotypic tissue culturing.

A) The two unilateral SCN nuclei (insert) dissected from the slice shown. B) Culture dish (35 mm Petri dish) with culture membrane, medium and explant, but without vacuum grease and cover glass. The medium $(1.2 \mathrm{~mL})$ can be seen as liquid between the dish and the membrane. One unilateral SCN nucleus is placed on the culture membrane. The whitest part of the small tissue is a piece of the optic chiasm (insert). C) The culture dish with its membrane and its SCN tissue, sealed with vacuum grease and a round cover glass. 


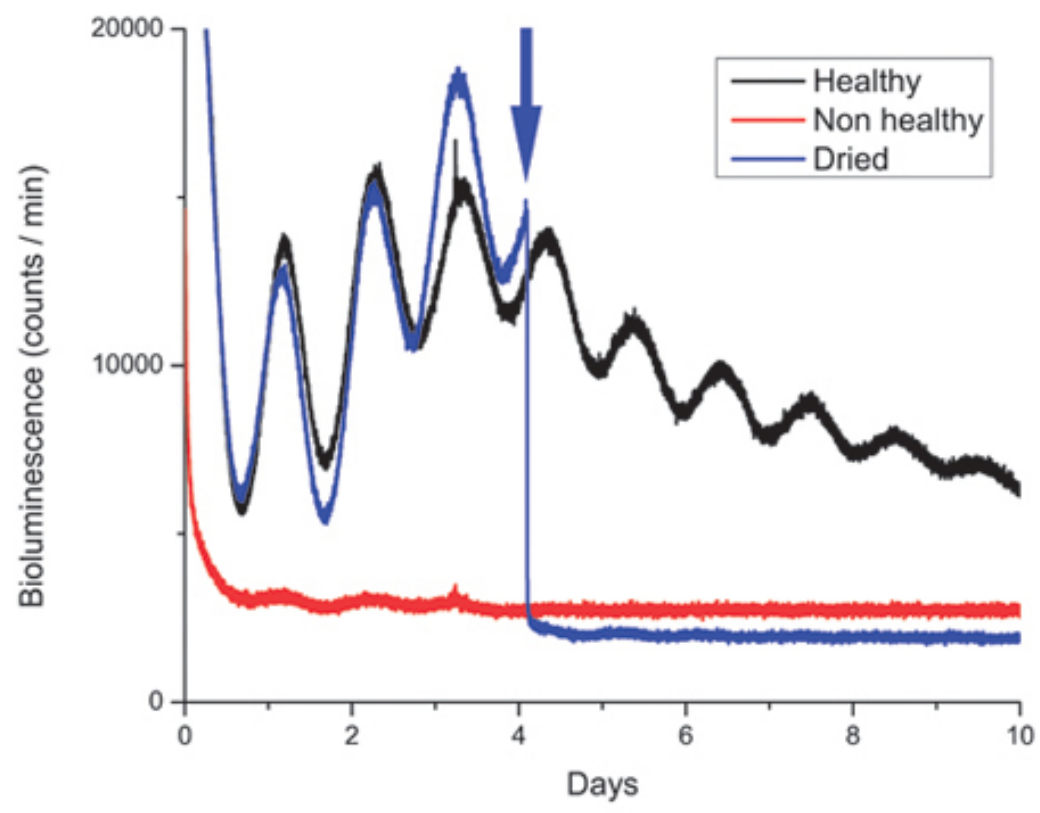

Figure 3. Bioluminescence recordings from healthy and non-healthy SCN tissue cultures.

Examples of bioluminescence recordings of PERIOD2::LUCIFERASE (PER2::LUC) expression in suprachiasmatic nucleus (SCN) slices obtained from mice held in a $12 \mathrm{~h}: 12 \mathrm{~h}$ light:dark cycle. The PER2::LUC protein oscillates with a circadian ( $24 \mathrm{hr})$ variation in which the maximum expression of the protein occurs at Zeitgeber time 12-13. Thus, the phase of the gene rhythm is dependent on the light dark schedule in which the animal was kept before sacrificed. Typically, bioluminescence from unilateral SCN tissues dissected as described in the protocol shows photon counts between $\sim 10.000-40.000$ /minute. The figure shows traces from one healthy (black) SCN culture, one non-healthy SCN culture (red) and one SCN culture that dried out (blue) after opening the sealed culture dish at day 4 and not re-sealing the dish properly (indicated by arrow).

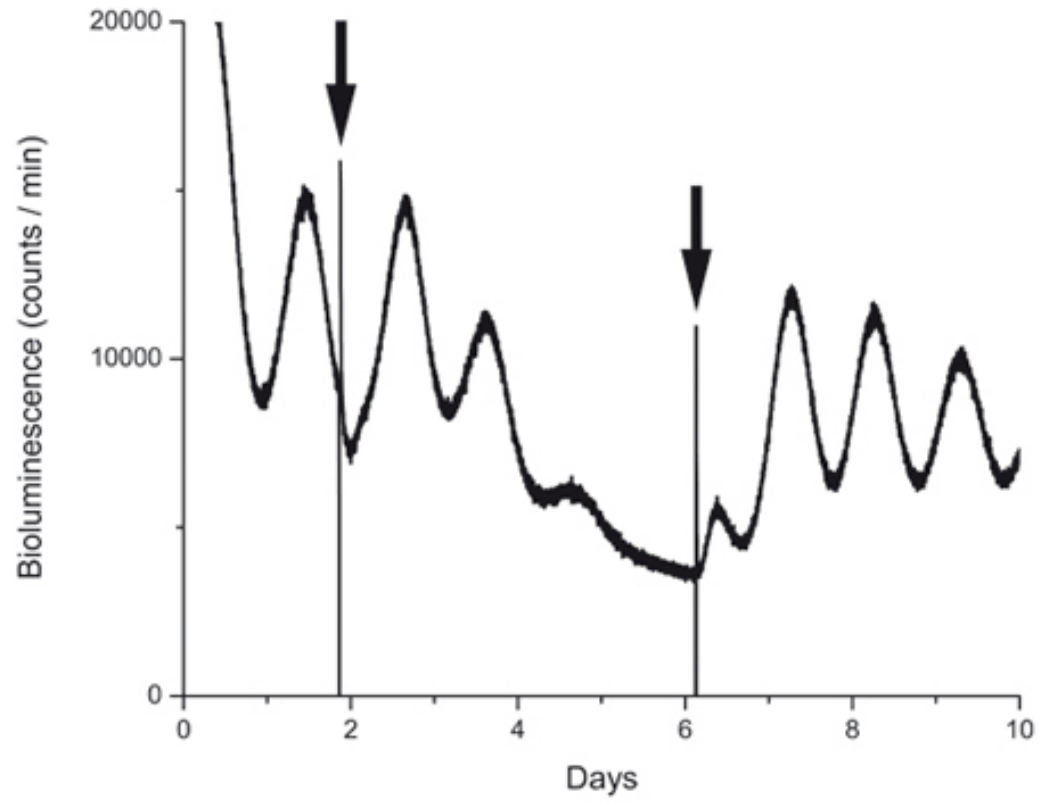

Figure 4. Bioluminescence recording during and after drug exposure.

PER2::LUC expression in a culture before, during and after action of a HCN channel blocker (ZD7288, $10 \mu \mathrm{M})$. The first arrow indicates the time when the blocker was added. The second arrow indicates washout, which was made by replacing the drug containing medium with conditioned control medium. Note the reduced amplitude after 2 days of drug exposure, lack of oscillation at day 4 and the quick recovery of the protein rhythm after washout. 


\section{Discussion}

\section{Benefits and disadvantages with luciferase reporter technology}

In contrast to ex vivo methods, such as RT-PCR, in situ hybridization and Western blot that require tissue sampling at many different time points (giving a low time resolution of normally $2-4 \mathrm{hrs}$ depending on sampling frequency) in order to study diurnal variations in gene and protein expressions, the luciferase reporter technology allows high resolution (1-10 min) studies of circadian oscillations for many days in the same preparation. Thus, the number of used animals is minimized and detailed studies of effects on phase and period of the rhythm are feasible, which normally is not possible using conventional sampling techniques with low time resolution. However, although relative amplitude measurements of the rhythm are possible, it should be emphasized that reporter technology is not quantitative and can therefore not be used to measure amounts of transcribed genes or translated proteins.

The tissue recordings can be started and analyzed immediately after culturing, a great advantage for directly studying molecular effects of in vivo stress, in vivo light-induced phase shifts etc. The organotypic SCN culture allows long-term (weeks) pharmacological manipulation of adult brain tissue containing an intact mature synaptic network and the luciferase reporter technology allows stable recordings for many weeks. Thus, the tissue does not need to be neonatal or early postnatal in order to obtain healthy cultures, and in contrast to the acute slice chronic pharmacological treatments can be performed. In contrast to plasmid-reporter transfections in cell cultures, which do no lead to incorporation of DNA into the genome, the transgenic luciferase-animal models (as well as lenti-virus transfections) are favorable if epigenetic alterations are to be studied. It should in this context also be mentioned that luminescence imaging is nowadays possible with highly sensitive CCD cameras ${ }^{11}$, allowing recordings even in single SCN neurons $(\sim 6-10 \mu \mathrm{m})$ and other cell types, utilized by major laboratories studying circadian rhythms. Finally, several circadian investigators commonly use monitoring of molecular expression using other reporters, such as the Green Fluorescent Protein, in order to study clock gene/protein oscillations ${ }^{16-19}$

\section{Aspects of slice thickness}

The here recommended thicknesses of the SCN slice $(200-300 \mu \mathrm{m})$ could be reduced or increased if desired. However, although the tissue flattens out on the membrane after a few days in culture, it is not recommended to exceed $500 \mu \mathrm{m}$ thickness of the initially cut slice in order to preserve viability of the tissue ${ }^{7}$. A slice thinner than $100 \mu \mathrm{m}$ is of mechanical and practical reasons difficult to handle. Because the SCN tissue is heterogeneous the thickness of the slice may affect the phase of the luciferase output signal, since different regions within the SCN (for instance the "core" versus the "shell", and dorsal versus ventral regions) oscillate with different phases ${ }^{20}$ and differentially re-synchronize after phase shifts ${ }^{21}$. The thickness of the slice also affects the baseline of photon count since more tissue emits a larger number of photons. The amplitude of molecular oscillations may in this way indirectly be affected by the size of the explant. For these reasons, control and treated cultures should always be of the same size, thickness and containing the same region of SCN in order to oscillate in the same phase and with the same amplitude. The two unilateral nuclei, on the other hand, oscillate in phase with each other and with similar amplitudes as long as the vibratome cut is horizontal and not angled (which in turn is dependent on that the separation cut between cerebellum and the two hemispheres is perpendicular to the table surface). An investigator who performs SCN culturing might experience that SCN cultures do not oscillate in phase with each other. Practicing and standardization of the slicing technique, i.e. the slices are always cut at the same rostro-caudal level of the SCN, will improve the outcome.

\section{Critical steps}

1. Oxygen supply is critical.

Because the brain requires a lot of oxygen ${ }^{22}$, the slicing procedure needs to be quick (The closer 3.1-3.10 is to $\sim 5-6$ minutes the better it is in order to keep the tissue healthy).

2. The SCN is sensitive to temperature changes and medium exchange.

The SCN is temperature sensitive and big temperature changes can phase shift the pacemaker and cause experimental artifacts ${ }^{23}$. If medium or solutions are exchanged or added during an ongoing experiment, for example when applying a drug after a couple of days in culture, the medium or solution needs to be pre-warmed to $36-37^{\circ} \mathrm{C}$ (the same temperature as in the chamber) before addition. In pharmacological experiments that involve medium exchanges, it is important to always work with pre-conditioned cell culture medium. Addition/wash-out with fresh, unconditioned medium can potentially phase shift the molecular SCN rhythm ${ }^{24}$ as well as other cell type cultures $^{25}$.

3. Medium composition.

It is important to culture the tissue in correct $\mathrm{pH}$ and osmolality. The air-buffering medium contains a large amount of HEPES, which has an optimal buffer capacity in the range of $\mathrm{pH} \mathrm{6.8-8.2} \mathrm{and} \mathrm{is} \mathrm{also} \mathrm{appropriate} \mathrm{for} \mathrm{buffering} \mathrm{the} \mathrm{pH}$ changes that may occur as a result of cell respiration. HEPES, on the other hand, is more sensitive to temperature changes as compared with sodium bicarbonate, which is included in small amounts in the culture medium. Sodium bicarbonate has a greater buffering capacity in the lower $\mathrm{pH}(5.1-7.1)$ range ${ }^{26}$, thus appropriate in $\mathrm{CO}_{2}$ atmosphere. However, increased amounts of sodium bicarbonate in the air-buffering medium (DMEM 2902) increases the osmolality significantly and can not be added without simultaneously diluting the medium, which in turn results in the incorrect composition of amino acids, vitamins and ions.

Advice for preparing the medium:

1. Be precise and careful when mixing the medium.

2. Use only fresh or freshly thawed stock solutions.

3. In case of very high osmolality it is not worth diluting. More than 5-6\%-diluted medium will most likely not work. Use D-glucose to increase osmolality if it is too low.

4. Phase shifts due to preparation time 
The time of slice preparation is critical. Zero or minimal effect on phase is obtained if the slice procedure is performed between $\mathrm{ZT} 6-12{ }^{12}$. All slice dissections should be performed at the same phase of the diurnal or circadian cycle in order to minimize error due to phase variation between the preparations.

\section{Possible modifications}

1. For acute SCN electrophysiology, the slicing has to be performed with a Ringer (Artificial Cerebro Spinal Fluid; ACSF) buffer, saturated with oxygen $\left(95 \% \mathrm{O}_{2}, 5 \% \mathrm{CO}_{2}\right)$ before the slicing starts. Further, oxygen needs to be added continuously to the buffer during the entire slicing procedure, as the electrical and synaptic activity is directly dependent on oxygen supply ${ }^{22}$.

2. Horizontal and sagittal slices can also be successfully prepared and cultured of the SCN. The authors have experience with luciferase recordings in horizontal slices, which according to our experience give circadian oscillations with lower amplitude as compared with the coronal cut.

3. The SCN is approximately $1 \mathrm{~mm}$ long rostro-caudally and the slicing technique described here is targeted towards acquiring an SCN section in the central region. However, more than one SCN section can be obtained from mouse and rat brains, allowing the investigation of circadian gene/protein expression at more rostral versus caudal levels of the SCN nucleus.

4. Slice cultures from younger animals, postnatal pups or even embryos, can also be prepared. Please note that the brain and the skull in very young pups are very soft and sensitive. In addition, the optic nerve is thin and not fully developed. Take caution when dissecting the brain of pups so that the SCN region is not damaged. For instance, micro rongeur tools cannot be used to open the skull of mouse pups because they are too big. Fine scissors are enough to cut the soft tissues in young pups.

5. The cover glass based culture technique described here can also be used to image luminescence using a CCD/EM-CCD camera, also in single SCN neurons.

6. Other central nervous system regions and peripheral organ tissues from transgenic PER2::LUC animals can easily be obtained, cultured and analyzed in terms of molecular oscillations, as the PER2::LUC expression continues to oscillate for many days also in a number of other tissues and cell types ${ }^{10}$. Most of these peripheral tissues, for instance liver, do not require a membrane for survival but are instead cultured directly bathed in the medium ${ }^{11}$. Please note that phase shifting due to sectioning, culturing and medium exchange may not follow the same principles as for SCN.

\section{Significance}

In transgenic mouse and rat strains (mPER2::LUC; mPer1-luc ${ }^{8,10,27}$ ) luciferase enzyme activity reflects protein or gene expression rhythms and can be assessed by bioluminescence recording. The luciferase-generated bioluminescence gives a weak signal but the background luminescence is close to zero, making this method advantageous. Moreover, because the luciferase molecule is unstable and rapidly degraded there is no phototoxicity, which can appear during long-term excitatory illumination ${ }^{28}$. Taken together, these properties allow long-lasting experiments making the luciferase reporter technology highly advantageous in circadian research.

\section{Disclosures}

No conflicts of interest declared.

\section{Acknowledgements}

This work was funded by The Swedish Medical Research Council (K2009-75SX-21028-01-3, K2008-61X-20700-01-3); FONCICYT 000000000091984; the foundations of Jeansson, Söderström Königska sjukhemmet, Märtha Lundqvist and Sigurd och Elsa Goljes Minne; and Swedish Society of Medicine SLS-95151. Professor Gene D. Block, UCLA, is gratefully acknowledged for valuable comments on the manuscript. We thank Dr Michael Andäng and Dr Helena Johard for the HCN channel blocker, and Prof. Abdel El-Manira for providing video microscope.

\section{Ethical considerations:}

All experiments on animals were performed in accordance with the guidelines and regulations set forth by Karolinska Institutet and "Stockholm's Norra Djurförsöksetiska Nämnd". All animal experiments are performed with the intention to minimize any possible stress or discomfort to the animal.

\section{References}

1. Welsh, D.K., Takahashi, J.S., Kay, S.A. Suprachiasmatic nucleus: cell autonomy and network properties. Annu Rev Physiol $72: 551-577$ (2010).

2. Green, D.J., Gillette, R. Circadian rhythm of firing rate recorded from single cells in the rat suprachiasmatic brain slice. Brain Res 245 : 198-200 (1982).

3. Shibata, S., Oomura, Y., Kita, H., Hattori, K. Circadian rhythmic changes of neuronal activity in the suprachiasmatic nucleus of the rat hypothalamic slice. Brain Res 247 : 154-158 (1982).

4. Groos, G., Hendriks, J. Circadian rhythms in electrical discharge of rat suprachiasmatic neurones recorded in vitro. Neurosci Lett 34 : 283-288 (1982).

5. Moore, R.Y. The suprachiasmatic nucleus and the circadian timing system. In: Suprachiasmatic nucleus--The mind's clock (Klein, D., Moore, R., Reppert, S., eds), 13-15. New York: Oxford University Press (1991).

6. Van den Pol, A.N. The hypothalamic suprachiasmatic nucleus of rat: intrinsic anatomy. J Comp Neurol 191: 661-702 (1980).

7. Stoppini, L., Buchs, P.A., Muller, D. A simple method for organotypic cultures of nervous tissue. J Neurosci Methods 37 : 173-182 (1991).

8. Yamazaki, S., Numano, R., Abe, M., Hida, A., Takahashi, R., Ueda, M., Block, G.D., Sakaki, Y., Menaker, M., Tei, H. Resetting central and peripheral circadian oscillators in transgenic rats. Science $288: 682-685(2000)$. 
9. Geusz, M.E., Fletcher, C., Block, G.D., Straume, M., Copeland, N.G., Jenkins, N.A., Kay, S.A., Day RN. Long-term monitoring of circadian rhythms in c-fos gene expression from suprachiasmatic nucleus cultures. Curr Biol 7 : 758-766 (1997).

10. Yoo, S.H, Yamazaki, S., Lowrey, P.L., Shimomura, K.,, Ko, C.H., Buhr, E.D., Siepka, S.M., Hong, H.K., Oh, W.J., Yoo, O.J., Menaker, M., Takahashi, J.S. PERIOD2::LUCIFERASE real-time reporting of circadian dynamics reveals persistent circadian oscillations in mouse peripheral tissues. Proc Natl Acad Sci U S A 101 : 5339-5346 (2004).

11. Yamazaki, S., Takahashi, J.S. Real-time luminescence reporting of circadian gene expression in mammals. Methods Enzymol $393: 288-301$ (2005).

12. Yoshikawa, T., Yamazaki, S., Menaker, M. Effects of preparation time on phase of cultured tissues reveal complexity of circadian organization. J Biol Rhythms $20: 500-512$ (2005).

13. Franklin, K.B.J, Paxinos, G. The mouse brain in stereotaxic coordinates: Academic Press (1997).

14. Paxinos, G., Watson, C. The rat brain in stereotaxic coordinates, 4 Edition. San Diego: Academic Press (1998).

15. O'Neill, J.S., Maywood, E.S., Chesham, J.E., Takahashi, J.S., Hastings, M.H. cAMP-dependent signaling as a core component of the mammalian circadian pacemaker. Science 320 : 949-953 (2008).

16. Hughes, A.T., Guilding, C., Lennox, L., Samuels, R.E., McMahon, D.G., Piggins, H.D. Live imaging of altered period1 expression in the suprachiasmatic nuclei of Vipr2-/- mice. J Neurochem 106 : 1646-1657 (2008).

17. Zhang, D.Q., Zhou, T., Ruan, G.X., McMahon, D.G. Circadian rhythm of Period1 clock gene expression in NOS amacrine cells of the mouse retina. Brain Res $1050: 101-109$ (2005).

18. Quintero, J.E., Kuhlman, S.J., McMahon, D.G. The biological clock nucleus: a multiphasic oscillator network regulated by light. J Neurosci $23: 8070-8076$ (2003).

19. LeSauter, J., Yan, L., Vishnubhotla, B., Quintero, J.E., Kuhlman, S.J., McMahon, D.G., Silver, R. A short half-life GFP mouse model for analysis of suprachiasmatic nucleus organization. Brain Res $964: 279-287$ (2003).

20. Nakamura, W., Yamazaki, S., Takasu, N.N., Mishima, K., Block, G.D. Differential response of Period 1 expression within the suprachiasmatic nucleus. J Neurosci $25: 5481-5487$ (2005)

21. Davidson, A.J., Yamazaki, S., Arble, D.M., Menaker, M., Block, G.D. Resetting of central and peripheral circadian oscillators in aged rats. Neurobiol Aging $29: 471-477$ (2008).

22. Croning, M.D., Haddad GG. Comparison of brain slice chamber designs for investigations of oxygen deprivation in vitro. J Neurosci Methods $81: 103-111(1998)$

23. Burgoon, P.W., Boulant, J.A. Temperature-sensitive properties of rat suprachiasmatic nucleus neurons. Am J Physiol Regul Integr Comp Physiol 281 : R706-715 (2001).

24. Nishide, S.Y., Honma, S., Honma, K. The circadian pacemaker in the cultured suprachiasmatic nucleus from pup mice is highly sensitive to external perturbation. Eur J Neurosci $27: 2686-2690$ (2008).

25. Yoshikawa, T., Sellix, M., Pezuk, P., Menaker, M. Timing of the ovarian circadian clock is regulated by gonadotropins. Endocrinology 150 : 4338-4347 (2009).

26. Kohn, R.A., Dunlap, T.F. Calculation of the buffering capacity of bicarbonate in the rumen and in vitro. J Anim Sci 76 : 1702-1709 (1998).

27. Wilsbacher, L.D., Yamazaki, S., Herzog, E.D., Song, E.J., Radcliffe, L.A., Abe, M., Block, G., Spitznagel, E., Menaker, M., Takahashi JS. Photic and circadian expression of luciferase in mPeriod1-luc transgenic mice invivo. Proc Natl Acad Sci U S A 99 : $489-494$ (2002).

28. Sellix, M.T., Currie, J., Menaker, M., Wijnen, H. Fluorescence/luminescence circadian imaging of complex tissues at single-cell resolution. J Biol Rhythms $25: 228-232$ (2010). 